Pacific Journal of Mathematic 


\title{
THE SYMPLECTIC GROUP OVER A RING WITH ONE IN ITS STABLE RANGE
}

\author{
B. KIRKWOOD AND B. R. MCDONALD
}

\begin{abstract}
In this paper we determine the transitivity properties, generators and commutator subgroups of the symplectic group and its congruence subgroups over a commutative ring having one in its stable range and 2 unit.
\end{abstract}

1. Introduction. Much of the classical theory of the symplectic group over a field has been generalized to symplectic groups where the scalar ring is local. The papers [6], [8], [11], [12], [17], [18] and [19] contain much of this literature and an introduction is provided in the monograph [13].

When using the local ring the technique is often to either "lift" results from the symplectic group over the residue class field or to utilize the abundance of units in local ring and mimic the arguments over a field.

However, the key to much of this theory is the ability to write units in the ring in a linear or polynomial fashion. This idea was exploited in [14] and several subsequent papers on the orthogonal group. In this paper, we show that the basic theory of the symplectic group over a commutative ring is available if the ring has "one in its stable range". This stable range condition is defined and discussed in (II). Examples of rings with one in their stable range include local rings, semilocal rings, von Neumann regular rings and zero dimensional rings.

The approach which allows this generalization is the extensive use of the "Eichler-Siegel-Dickson transvections" rather than the more traditional "symplectic transvections". In a sense, this "linearizes" the theory, allowing arguments which resemble the general linear group and elementary transvections. If $R$ has one in its stable range, then utilizing repeatedly the formulas $\left(^{*}\right)$ and $\left.{ }^{* *}\right)$ we create units in desired locations. Once these units are available, the standard results easily follow. It should be emphasized that the theory we present is a consequence of formulas $\left(^{*}\right)$ and $\left(^{* *}\right)$.

2. The symplectic group. Let $R$ denote a commutative ring. We let $V$ be a free $R$-module of $R$-dimension $n$ where $n \geqq 2$. We assume $V$ has a nonsingular symplectic form $\beta: V \times V \rightarrow R$. That 
is, $\beta$ is $R$-bilinear, $\beta(x, x)=0$ for all $x$ in $V$ and the $R$-module morphism from $V$ to $V^{*}=\operatorname{Hom}_{R}(V, R)$ given by $x \rightarrow \beta(, x)$ is an isomorphism. We call the pair $(V, \beta)$ a symplectic space. When the context is clear, $(V, \beta)$ will be denoted by $V$.

Recall that an element $x$ in $V$ is unimodular if there is an $f$ in $V^{*}$ with $f(x)=1$; equivalently, if $x=\alpha_{1} b_{1}+\cdots+\alpha_{n} b_{n}$ where $\left\{b_{1}, \cdots, b_{n}\right\}$ is a basis for $V$, then $x$ is unimodular if $\left(\alpha_{1}, \cdots, \alpha_{n}\right)=R$. If $x$ is unimodular then $R x$ is a free $R$-direct summand of dimension one. We call $R x$ a line. If $x$ is unimodular and $V=R x \oplus W$, we call the projective module $W$ a hyperplane. Locally, $W$ will have dimension $n-1$, but $W$ need not be free.

A hyperbolic pair $\{x, y\}$ is a pair of unimodular vectors in $V$ with the property that $\beta(x, y)=1$. The module $H=R x \oplus R y$ is called a hyperbolic plane and it is easy to see (for example, see [13], pp. 150-151) that $V$ splits as an orthogonal direct sum $V=H \perp H^{\perp}$ where $H^{\perp}$ denotes the orthogonal complement of $H$.

Any unimodular vector $u$ may be complemented to a hyperbolic pair as follows: By the above comments, there is an $f$ in $V^{*}$ with $f(u)=1$. Since $\beta$ is nonsingular, there is a $v$ in $V$ with $1=f(u)=$ $\beta(u, v)$. Then $\{u, v\}$ is a hyperbolic pair.

A ring $R$ is stably free if whenever $V=V_{1} \oplus P$ where $V$ and $V_{1}$ are free $R$-modules, then $P$ is a free $R$-module. Combining this with the above remarks on hyperbolic pairs, we have the following proposition.

Proposition 2.1. Let $R$ be a stably free commutative ring and $V$ be a symplectic space over $R$. Then $V$ is an orthogonal direct sum $V=H_{1} \perp H_{2} \perp \cdots \perp H_{m}$ of hyperbolic planes $H_{1}, H_{2}, \cdots, H_{m}$. In particular, the dimension of $V$ is even.

Let $(V, \beta)$ and $(\bar{V}, \bar{\beta})$ be symplectic spaces of the same dimension. An $R$-module isomorphism $\sigma: V \rightarrow \bar{V}$ is an isometry if for all $x$ and $y$ in $V$, we have $\bar{\beta}(\sigma(x), \sigma(y))=\beta(x, y)$. In this case we say $V$ and $\bar{V}$ are $i$ sometric, denoted $V \simeq \bar{V}$. The group of isometries $\sigma:(V, \beta) \rightarrow$ $(V, \beta)$ is called the symplectic group of $V$ and denoted by $\operatorname{Sp}(V)$.

Suppose $V=H \perp W$ where $H=R u \oplus R v$ is a hyperbolic plane in $V$. We next define several standard isometries with respect to $H$. 
(a) If $x$ is in $V$ with $\beta(x, u)=0$, then the Eichler-Siegel-Dickson transvection (denoted ESD-transvection) $\sigma_{u, x}$ is given by

$$
\sigma_{u, x}(y)=y+\beta(u, y) x+\beta(x, y) u .
$$

If $\beta(x, v)=0$, then $\sigma_{v, x}$ is defined in a similar fashion.

(b) If $\varepsilon$ is a unit in $R$, then define the isometry $\Phi_{\varepsilon}$ by

$$
\Phi_{\varepsilon}(u)=\varepsilon u, \quad \Phi_{\varepsilon}(v)=\varepsilon^{-1} v,
$$

and $\Phi_{\varepsilon}(w)=w$ for all $w$ in $W$.

(c) Define the isometry $\Delta$ by $\Delta(u)=v, \Delta(v)=-u$ and $\Delta(w)=w$ for all $w$ in $W$.

It is straightforward to check that the above are isometries. (Note that $\beta(\sigma(x), \sigma(y))=\beta(x, y)$ may be checked locally since each of the above localize nicely at prime ideals of $R$ and here one may use ([13], pp. 159-161).)

A symplectic transvection $\tau$ is an isometry satisfying any of the following equivalent statements:

(a) There is a unimodular vector $a$ in $V$ and a scalar $\lambda$ in $R$ such that for all $x$ in $V$,

$$
\tau(x)=x+\lambda \beta(a, x) a .
$$

(b) There is a line $L=R a$ satisfying $\tau(x)-x$ is in $L$ for all $x$ in $V$.

(c) There is a hyperplane $P$ with $\left.\tau\right|_{P}=$ identity. (See Theorem 4.1 , p. 191 of [13].)

The above symplectic transvection is denoted by $\tau_{a, \lambda}$ and we call $L=R a$ the line of $\tau_{a, \lambda}$ and $P$ the hyperplane of $\tau_{a, \lambda}$.

The basic calculational properties of the above isometries are summarized in the next lemma.

LEMMA 2.2. Let $R$ be a commutative ring. Let $V$ be a symplectic space over $R$ with $V=H \perp W$ where $H=R u \oplus R v$ is a hyperbolic plane. Then

(a) $\sigma_{u, x} \sigma_{u, y}=\sigma_{u, x+y},\left(\sigma_{u, x}\right)^{-1}=\sigma_{u,-x}$, and $\sigma_{u, 0}=I$.

(b) If $\theta$ is in $\operatorname{Sp}(V)$ then $\theta \sigma_{u, x} \theta^{-1}=\sigma_{\theta(u), \theta(x)}$.

(c) $\Delta^{-1} \Phi_{\varepsilon} \Delta=\Phi_{\varepsilon^{-1}}=\Phi_{\varepsilon}^{-1}, \quad \Phi_{\varepsilon} \Delta \Phi_{\varepsilon}=\Delta, \Delta^{4}=I$, and $\Phi_{\varepsilon} \sigma_{u, x} \Phi_{\varepsilon}^{-1}=\sigma_{u, \varepsilon x}$ when $x$ is in $W$.

(d) If $x=\alpha u+\bar{x}$ and $y=\delta u+\eta v+\bar{y}$ where $\alpha, \delta$, $\eta$ are in $R$ and $\bar{x}, \bar{y}$ are in $W$, then 


$$
\sigma_{u, x}(y)=[\delta+2 \alpha \eta+\beta(\bar{x}, \bar{y})] u+\eta v+(\bar{y}+\eta \bar{x}) .
$$

If $x=\alpha v+\bar{x}$ and $y=\delta u+\eta v+\bar{y}$ with the same hypothesis as above, then

$$
\sigma_{v, x}(y)=\delta u+[\eta-2 \alpha \delta+\beta(\bar{x}, \bar{y})] v+(\bar{y}-\delta \bar{x}) .
$$

(e) $\tau_{a, \lambda}^{-1}=\tau_{a,-\lambda}, \tau_{a, \lambda} \tau_{a, \mu}=\tau_{a, \lambda+\mu}$ and, more generally, $\tau_{a, \lambda} \tau_{b, \mu}(x)=$ $x+[\lambda \beta(a, x) a+\mu \beta(b, x) b]+\lambda \mu \beta(a, b) \beta(b, x) a$.

(f) If $\theta$ is in $\operatorname{Sp}(V)$, then $\theta \tau_{a, \lambda} \theta^{-1}=\tau_{\theta(a), \lambda}$.

(g) The above isometries may be written as symplectic transvections and ESD-transvections as follows:

$$
\begin{aligned}
\Phi_{\varepsilon} & =\tau_{u, \varepsilon(\varepsilon-1)} \tau_{v, \varepsilon-1(\varepsilon-1)} \tau_{u+v, 1-\varepsilon} \\
\Delta & =\tau_{v,-2} \tau_{u-v,-1} \\
\tau_{a, \lambda} & =\sigma_{a,(\lambda / 2) a} \text { (if } 2 \text { is a unit). }
\end{aligned}
$$

Thus, each of the above isometries may be written as products of ESD-transvections if 2 is a unit in $R$. Also, an argument analogous to the discussion in ([13], pp. 193-197) shows that each ESD-transvection may be written as a product of symplectic transvections.

THEOREM 2.3. Let $R$ be a commutative ring having 2 a unit. Let $V$ be a symplectic space over $R$ with $V=H \perp W$ where $H=$ $R u \oplus R v$ is a hyperbolic plane. Suppose $\sigma$ is in $\operatorname{Sp}(V)$. If $\sigma(v)=$ $\alpha u+\delta v+t(t$ in $W)$ and $\delta$ is a unit, then $\sigma$ may be written as

$$
\sigma=\sigma_{u, x} \sigma_{v, y} \Phi_{\varepsilon} \bar{\sigma}
$$

where $\bar{\sigma}$ is in $\mathrm{Sp}(W)$. Further, $x$ is in $(R u)^{\perp}, y$ is in $(R v)^{\perp}$, and $x, y, \varepsilon$ and $\bar{\sigma}$ are uniquely determined by $\sigma$.

(Note: We identify $\operatorname{Sp}(W)$ as a subgroup of $\operatorname{Sp}(V)$ by $\bar{\sigma} \rightarrow$ $I \perp \bar{\sigma}$.

Proof. Suppose $\sigma(v)=\alpha u+\delta v+t$ where $\delta$ is a unit. Then

$$
\begin{aligned}
\sigma_{u, \delta^{-1} t \delta^{-1}(\alpha / 2) u}(v) & =v+\delta^{-1} t+\delta^{-1} \alpha u \\
& =\delta^{-1}[\alpha u+\delta v+t] \\
& =\delta^{-1} \sigma(v) .
\end{aligned}
$$

Thus, set

$$
\hat{\sigma}=\Phi_{\delta} \sigma_{u, \delta^{-1} t^{-1}{ }^{-1}(\alpha / 2) u}
$$

and we have $\hat{\sigma}(v)=v$. Suppose $\hat{\sigma}(u)=\gamma(u)+\mu v+s(s$ in $W)$. Since $\beta(u, v)=1$, we have $\hat{\sigma}(u)=u+\mu v+s$. Then 


$$
\begin{aligned}
& \sigma_{v,(\mu / 2) v+s}(\hat{\sigma}(u))=u \\
& \sigma_{v,(\mu / 2) v+s}(\hat{\sigma}(v))=v .
\end{aligned}
$$

That is,

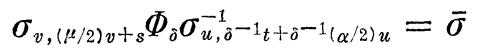

where $\left.\bar{\sigma}\right|_{H}=$ identity, i.e., $\bar{\sigma}$ is in $\operatorname{Sp}(W)$. Thus, using 2.2, $\sigma=$ $\sigma_{u, x} \sigma_{v, y} \Phi_{\varepsilon} \bar{\sigma}$ for suitable $x, y$ and $\varepsilon$.

It remains to check uniqueness. Assume

$$
\sigma_{u, x} \sigma_{v, y} \Phi_{\varepsilon} \bar{\sigma}=\sigma_{u, x_{1}} \sigma_{v, y_{1}} \Phi_{\varepsilon_{1}} \bar{\sigma}_{1}
$$

Apply both sides of the above equality to $v$. We obtain

$$
\varepsilon^{-1} \beta(x, v) u+\varepsilon^{-1} v+\varepsilon^{-1} x=\varepsilon_{1}^{-1} \beta\left(x_{1}, v\right) u+\varepsilon_{1}^{-1} v+\varepsilon_{1}^{-1} x_{1}
$$

which implies $\varepsilon=\varepsilon_{1}$. If $x=\alpha u+\bar{x}$ and $x_{1}=\alpha_{1} u+\bar{x}_{1}$ as in $2.2(\mathrm{~d})$, then

$$
\alpha u+v+(\alpha u+\bar{x})=\alpha_{1} u+v+\left(\alpha_{1} u+\bar{x}_{1}\right) .
$$

Then, since 2 is a unit we have $\alpha=\alpha_{1}$ and subsequently $\bar{x}=\bar{x}_{1}$. A similar argument utilizing $u$ will show $y=y_{1}$.

Suppose that $V$ splits as a direct sum of hyperbolic planes $V=$ $H_{1} \perp H_{2} \perp \cdots \perp H_{m}$ where $H_{i}=R u_{i} \oplus R v_{i}$ for $1 \leqq i \leqq m$. The basis $\left\{u_{1}, v_{1}, u_{2}, v_{2}, \cdots, u_{m}, v_{m}\right\}$ is called a hyperbolic basis of $V$.

Suppose we have the above hyperbolic basis for $V$. For $x$ and $z$ in $V$, let

$$
x=\sum_{i=1}^{m} \alpha_{i} u_{i}+\sum_{i=1}^{m} \gamma_{i} v_{i},
$$

and

$$
z=\sum_{i=1}^{m} \delta_{i} u_{i}+\sum_{i=1}^{m} \eta_{i} v_{i}
$$

If $\beta\left(x, u_{1}\right)=0$, i.e., $\gamma_{1}=0$, then

$$
\begin{aligned}
\sigma_{u_{1}, x}(z)= & {\left[\delta_{1}+2 \alpha_{1} \eta_{1}+\sum_{i=2}^{m}\left(\alpha_{i} \eta_{i}-\delta_{i} \gamma_{i}\right)\right] u_{1} } \\
& +\eta_{1} v_{1}+\sum_{i=2}^{m}\left[\left(\delta_{i}+\eta_{1} \alpha_{i}\right) u_{i}+\left(\eta_{i}+\eta_{1} \gamma_{i}\right) v_{i}\right] .
\end{aligned}
$$

If $\beta\left(x, v_{1}\right)=0$, i.e., $\alpha_{1}=0$, then 


$$
\begin{aligned}
\sigma_{v_{1}, x}(z)= & \delta_{1} u_{1}+\left[\eta_{1}-2 \delta_{1} \gamma_{1}+\sum_{i=2}^{m}\left(\alpha_{i} \eta_{i}+\gamma_{i} \delta_{i}\right)\right] v_{1} \\
& +\sum_{i=2}^{m}\left[\left(\delta_{i}-\delta_{1} \alpha_{i}\right) u_{i}+\left(\eta_{i}-\delta_{1} \gamma_{i}\right) v_{i}\right] .
\end{aligned}
$$

A commutative ring $R$ is said to have one in its stable range or have stable range one if whenever $\alpha$ and $\beta$ are in $R$ with $(\alpha, \beta)=R$ then there is a $\delta$ in $R$ with $\alpha+\delta \beta=$ unit. Stable range one rings (both commutative and noncommutative) have been examined from a ring theoretic viewpoint in [3], [4], [5], and [20]. The role that stable range one rings play in linear algebra and the general linear group is discussed in [1], [4], [7], [15], [21], [22], [23], and [24]. This definition was extended in [9], [10], [14], and [16] to examine the structure theory of quadratic forms, Witt rings and the orthogonal group. In particular, in ([14], 3.1) it was noted that a ring having stable range one was stably free and, hence, for our purposes their symplectic spaces are direct sums of hyperbolic planes. Examples of rings with one in their stable range are local rings, semilocal rings, von Neumann regular rings, and zero dimensional rings.

Suppose $R$ has 2 a unit and stable range one. It is straightforward to show that if $\left(x, y_{1}, y_{2}, \cdots, y_{n}\right)=R$ then there are $\alpha_{1}$, $\alpha_{2}, \cdots, \alpha_{n}$ in $R$ with

$$
x+\alpha_{1} y_{1}+\cdots+\alpha_{n} y_{n}=\text { unit . }
$$

Returning to the above calcutation $\left(^{*}\right)$ (or $\left(^{* *}\right)$ ) suppose that $z=\sum_{i} \delta_{i} u_{i}+\sum_{i} \eta_{i} v_{i}$ is unimodular. Since 2 is a unit, we then have

$$
\left(\delta_{1}, 2 \eta_{1},-\delta_{2}, \eta_{2}, \cdots,-\delta_{m}, \eta_{m}\right)=R .
$$

Thus, there exist $\alpha_{1}, \alpha_{2}, \cdots, \alpha_{m}, \gamma_{2}, \gamma_{3}, \cdots, \gamma_{m}$ with

$$
\delta_{1}+2 \alpha_{1} \eta_{1}+\sum_{i}\left(\alpha_{i} \eta_{i}-\gamma_{i} \delta_{i}\right)=\text { unit . }
$$

That is, if $x=\alpha_{1} u_{1}+\cdots+\alpha_{m} u_{m}+\gamma_{2} v_{2}+\cdots+\gamma_{m} v_{m}$ then

$$
\sigma_{u_{1}, x}(z)=\delta u_{1}+\eta v_{1}+\bar{z}
$$

where $\delta$ is a unit.

We will now develop a number of consequences of the above observation. First, is a sharpening of 2.3.

THEOREM 2.4. Let $R$ be a commutative ring with stable range one and 2 a unit. Let $V=H \perp W$ be a symplectic space over $R$ where $H=R u \oplus R v$ is a hyperbolic plane. Let $\sigma$ be in $\operatorname{Sp}(V)$. 
Then there is a $z$ in $V$ such that

$$
\sigma=\sigma_{u, z} \sigma_{u, x} \sigma_{v, y} \Phi_{\varepsilon} \bar{\sigma}
$$

where $x$ is in $(R u)^{\perp}, y$ is in $(R v)^{\perp}, \varepsilon$ is a unit, and $\bar{\sigma}$ is in $\operatorname{Sp}(W)$ and each is uniquely determined by $\sigma$ and $z$.

Proof. Consider the unimodular vector $\sigma(v)$. By the discussion before 2.4, there is an ESD-transvection $\sigma_{u, z}$ with

$$
\left(\sigma_{u, z} \sigma\right)(v)=\sigma_{u, z}(\sigma(v))=\alpha u+\delta v+t(t \text { in } W)
$$

where $\delta$ is a unit. The result now follows from 2.3.

Let $H=R u \oplus R v$ be a hyperbolic plane. Let $E(u, v)$ denote the subgroup of $\operatorname{Sp}(V)$ generated by the isometries of the form $\sigma_{u, x}$ and $\sigma_{v, y}$ for suitable $x$ and $y$. Let $P(u, v)$ denote the group generated by the $\Phi_{\varepsilon}$ for $\varepsilon$ a unit. Finally, let $E(H)$ denote the subgroup generated by all ESD-transvections $\sigma_{a, x}$ and $\sigma_{b, y}$ where $H=R a \oplus R b$ and $\beta(a, b)=1$.

COROLLARY 2.5 (under the hypothesis of 2.4).

(a) $\operatorname{Sp}(V)=E(u, v) P(u, v) \operatorname{Sp}(W)$.

(b) $\operatorname{Sp}(V)=E(H) \operatorname{Sp}(W)$.

Proof. Part (a) follows immediately from 2.4. Part (b) follows from 2.4 and part $(\mathrm{g})$ of 2.2 which shows each $\Phi_{\varepsilon}$ may be written as a product of elements in $E(H)$.

COROLLARY 2.6 (under the hypothesis of 2.4). The group $E(H)$ is a normal subgroup of $\operatorname{Sp}(V)$.

Proof. Let $\tau=\sigma_{u, x}$ be in $E(H)$. Let $\rho$ be in $\operatorname{Sp}(V)$. By 2.5, $\rho=\sigma \bar{\rho}$ where $\sigma$ is in $E(H)$ and $\bar{\rho}$ is $\operatorname{in} \operatorname{Sp}(W)$. Then

$$
\begin{aligned}
\rho \tau \rho^{-1} & =(\sigma \bar{\rho})\left(\sigma_{a, x}\right)(\sigma \bar{\rho})^{-1} \\
& =\sigma\left(\bar{\rho} \sigma_{u, x} \bar{\rho}^{-1}\right) \sigma^{-1} \\
& =\sigma \sigma_{u, \bar{o}(x)} \sigma^{-1} \quad(\text { by 2.2(b)) }
\end{aligned}
$$

and this final product is in $E(H)$.

Under the hypothesis of 2.4, the symplectic space $V$ splits as an orthogonal sum $V=H_{1} \perp \cdots \perp H_{m}$ where the $H_{i}$ are hyperbolic planes. On the other hand $V=H_{1} \perp W$. Since orthogonal complements are unique, $W=H_{2} \perp \cdots \perp H_{m}$. An induction argument will now give the first part of the following corollary. 
COROLLARY 2.7 (under the hypothesis of 2.4). Suppose $V=H_{1} \perp$ $H_{2} \perp \cdots \perp H_{m}$ is a decomposition of $V$ into hyperbolic planes. Then

(a) $\operatorname{Sp}(V)=E\left(H_{1}\right) E\left(H_{2}\right) \cdots E\left(H_{m}\right)$. Thus, the symplectic group $\mathrm{Sp}(V)$ is generated by ESD-transvections.

(b) Each element in $\operatorname{Sp}(V)$ is a product of $\leqq 6 m$ ESD-transvections where $m=(\operatorname{dim} V) / 2$.

Proof. Part (a) is immediate. Part (b) follows from 2.4, 2.2(g) and induction.

We next develop some transitivity results related to the calculations $(*)$ and $(* *)$.

Let $R$ have stable range one and $V=H \perp W$ where $H=R u \oplus R v$ is a hyperbolic plane. If $z$ is a unimodular vector in $V$ then, by using (*) and the calculation prior to 2.4, there is an ESD-transvection $\sigma_{u, x}$ with

$$
\sigma_{u, x}(z)=\delta u+\eta v+\bar{z} \quad(\bar{z} \text { in } W)
$$

where $\delta$ is a unit. Let $y=\delta^{-1} \bar{z}$. Then

$$
\begin{aligned}
\sigma_{v, y} \sigma_{u, x}(z) & =\sigma_{v, y}[\delta u+\eta v+\bar{z}] \\
& =\delta u+\eta v+(\bar{z}-\delta y) \\
& =\delta u+\eta v .
\end{aligned}
$$

That is, $\sigma_{v, y} \sigma_{u, x}(z)$ is in the hyperbolic plane $H$.

THEOREM 2.8. Let $R$ be a commutative ring with stable range one and 2 a unit. Let $V$ be a symplectic space over $R$. Then,

(a) $\operatorname{Sp}(V)$ is transitive on unimodular vectors.

(b) $\operatorname{Sp}(V)$ is transitive on hyperbolic planes.

Proof. (a) Suppose $z$ and $\bar{z}$ are unimodular vectors in $V$. By the above discussion, there are products $\sigma_{1}$ and $\sigma_{2}$ of isometries in $\operatorname{Sp}(V)$ such that

$$
\begin{aligned}
& \sigma_{1}(z)=\delta u+\eta v \\
& \sigma_{2}(\bar{z})=\bar{\delta} u+\bar{\eta} v
\end{aligned}
$$

where $\delta, \bar{\delta}$ are units and $H=R u \oplus R v$ is a hyperbolic plane. Consider the vector $\sigma_{1}(z)=\delta u+\eta v$. Then $\Phi_{\delta^{-1}}\left(\sigma_{1}(z)\right)=u+\mu v$ where $\mu=\delta \eta$. Then, using transvection $\tau=\tau_{v+u+\mu_{v, 1}}$, we have

$$
\tau \bar{\Phi}_{\dot{\delta}^{-1}}\left(\sigma_{1}(z)\right)=-v \text {. }
$$

Similarly, a product of two isometries will carry $\bar{\delta} u+\bar{\eta} v$ to $-v$. That is, there are products $\Sigma_{1}$ and $\Sigma_{2}$ of isometries with 


$$
\sum_{1}(z)=-v=\sum_{2}(\bar{z}),
$$

i.e., $\sum_{2}^{-1} \sum_{1}(z)=\bar{z}$. This completes part (a). To show (b), let $\{u, v\}$ and $\{x, y\}$ be hyperbolic pairs. Since $u$ and $x$ are unimodular, there is a suitable product $\sigma$ of isometries so that $\sigma(u)=x$. Thus, without loss of generality, assume $u=x$. We need to carry $v$ to $y$ while fixing $u$. Let $y=\alpha u+\delta v+w$ where $w$ is in $(R u \oplus R v)^{\perp}$. Since $1=\beta(x, y)=\beta(u, y)=\delta$, we have $y=\alpha u+v+w$. Then

$$
\{u, y\} \underset{\sigma_{u,-w}}{\longrightarrow}\{u, \alpha u+v\} \underset{\tau_{u,-\alpha}}{\longrightarrow}\{u, v\},
$$

and we are done.

COROLlaRY 2.9 (cancellation). Let $R$ be a commutative ring having stable range one and 2 a unit. If $U, V$ and $Y$ are symplectic spaces with $U \perp V \simeq U \perp Y$, then $V \simeq Y$.

Proof. By an induction argument, it suffices to prove the result when $U=H$ is a hyperbolic plane. Let $\sigma: H \perp V \rightarrow H \perp Y$ be an isometry. Let $H_{1}=\sigma(H)$ and $V_{1}=\sigma(V)$. There is a product $\tau$ of elements in $\operatorname{Sp}(H \perp Y)$ with $\tau H_{1}=H$. Then $\tau H_{1}^{\perp}=H^{\perp}$, i.e., $\tau V_{1}=Y$. Thus $V \simeq Y$.

If $V$ is a symplectic space of dimension 2 over a commutative ring with stable range one, then $\operatorname{Sp}(V)$ is precisely the special linear group SL $(V)$ of $V$. In this case, the structure of $\mathrm{SL}(V)$ is given in [15].

Proposition 2.10 (under the hypothesis of 2.4). The center of $\mathrm{Sp}(V)$ is precisely

$\left\{\alpha I \mid \alpha\right.$ is in $R$ and $\left.\alpha^{2}=1\right\}$.

Proof. If $\operatorname{dim}(V)=2$, this is given in [15]. If $\operatorname{dim}(V) \geqq 3$, the proof is analogous to ([13], Thm. 3.22).

Let $A$ be an ideal of $R$. The natural ring morphism $\pi_{A}: R \rightarrow$ $R / A$ induces a surjective morphism $\pi_{A}: V \rightarrow V / A V$ of symplectic spaces where if $V=(V, \beta)$ then $(V / A V, \bar{\beta})$ is given by

$$
\bar{\beta}\left(\pi_{A} x, \pi_{A} y\right)=\pi_{A} \beta(x, y) .
$$

It is easy to see that $R / A$ has stable range one if $R$ has stable range one, e.g., see (Prop. 2.6(a) of [14]). In turn, $\pi_{A}$ induces a group morphism $\pi_{A}: \mathrm{Sp}(V) \rightarrow \mathrm{Sp}(V / A V)$ by

$$
\left(\pi_{A} \sigma\right)\left(\pi_{A} x\right)=\pi_{A}(\sigma(x)) .
$$


A splitting $V=H_{1} \perp \cdots \perp H_{m}$ of $V$ into hyperbolic planes induces a splitting of $V / A V=\bar{H}_{1} \perp \cdots \perp \bar{H}_{m}$ into hyperbolic planes where $\pi_{A} H_{i}=\bar{H}_{i}, 1 \leqq i \leqq m$. Each generator, i.e., ESD-transvection, in $E\left(\bar{H}_{i}\right)$ has a preimage under $\pi_{A}$ in $E\left(H_{i}\right)$. By 2.7 these elements generate $\operatorname{Sp}(V / A V)$. Thus, we have the following proposition.

Proposition 2.11 (under the hypothesis of 2.4). The group morphism $\pi_{A}: \operatorname{Sp}(V) \rightarrow \operatorname{Sp}(V / A V)$, where $A$ is an ideal, is surjective.

We now study the commutator subgroup of $\operatorname{Sp}(V)$. To achieve the expected results we will see that we need units $\varepsilon$ and $\eta$ in $R$ such that $\varepsilon-\eta=1$. If 3 is a unit in $R$, then $3-2=1$ and 3 and 2 will do. More generally, we can always assure this will happen if $R$ has "2-fold" stable range one. Precisely, $R$ has 2-fold stable range one if whenever $\left(a_{1}, b_{1}\right)=R$ and $\left(a_{2}, b_{2}\right)=R$ then there is an $\alpha$ with $a_{1}+\alpha b_{1}=$ unit and $a_{2}+\alpha b_{2}=$ unit. The concept of " $k$-fold stable range one" rings was introduced in [7]. Suppose $R$ has 2 -fold stable range one. Then, using $(1,1)=R$ and $(0,1)=R$ we can find $\eta$ with $1+\eta=\varepsilon$ (unit) and $\eta=$ unit. Thus, we have units $\varepsilon$ and $\eta$ with $\varepsilon-\eta=1$.

If $G$ is a group, denote the commutator subgroup of $G$ by $[G, G]$.

THEOREM 2.12 (under the hypothesis of 2.4). Suppose there exists units $\varepsilon$ and $\eta$ in $R$ with $\varepsilon-\eta=1$. Then

$$
\operatorname{Sp}(V)=[\operatorname{Sp}(V), \operatorname{Sp}(V)] .
$$

Proof. It suffices to show that each generator $\sigma_{u, x}$ or $\sigma_{v, x}$ of $\operatorname{Sp}(V)$ can be written as a commutator. Consider $\sigma_{u, x}$. Select units $\varepsilon$ and $\eta$ in $R$ with $\varepsilon-\eta=1$. Let $\alpha=\eta^{-1}$. Then

$$
\begin{array}{rlr}
{\left[\Phi_{\varepsilon}, \sigma_{u, \alpha x}\right]} & =\Phi_{\varepsilon} \sigma_{u, \alpha x} \Phi_{\varepsilon}^{-1} \sigma_{u, \alpha x}^{-1} \\
& =\sigma_{u, \varepsilon \alpha x} \sigma_{u,-\alpha x} & (\text { by 2.2) } \\
& =\sigma_{\left.u, \varepsilon_{\alpha}-\alpha\right) x} & (\text { by 2.2) } \\
& =\sigma_{u, x} . &
\end{array}
$$

The isometry $\sigma_{v, x}$ is handled similarly.

3. The congruence subgroups. Let $A$ be an ideal of the ring $R$. As noted in the previous section, the ring morphism $\pi_{A}: R \rightarrow R / A$ induces a group morphism $\pi_{A}: \operatorname{Sp}(V) \rightarrow \operatorname{Sp}(V / A V)$. The group morphism is in general not surjective; however, if $R$ has stable range one and 2 a unit then it is surjective by 2.11 . 
The general congruence subgroup of level $A$ is

$$
\operatorname{GSp}(V, A)=\pi_{A}^{-1} \quad(\operatorname{center}(\operatorname{Sp}(V / A V)))
$$

where $0 \varsubsetneqq A \varsubsetneqq R$. The special cases are

$$
\operatorname{GSp}(V, R)=\operatorname{Sp}(V), \quad \operatorname{GSp}(V, 0)=\operatorname{Center}(\operatorname{Sp}(V)) .
$$

If $0 \varsubsetneqq A \varsubsetneqq R$, then the special congruence subgroup of level $A$ is

$$
\begin{aligned}
\operatorname{SSp}(V, A) & =\operatorname{ker}\left(\pi_{A}\right) \\
& =\left\{\sigma \text { in } \operatorname{Sp}(V) \mid \pi_{A} \sigma=I\right\} .
\end{aligned}
$$

The special cases are

$$
\operatorname{SSp}(V, R)=\operatorname{Sp}(V), \quad \operatorname{SSp}(V, 0)=\{I\} .
$$

If $\alpha$ is in $R$, then the order of $\alpha$, denoted $O(\alpha)$, is the ideal generated by $\alpha$. If $x$ is in $V$, then the order $O(x)$ of $x$ is the smallest ideal $A$ of $R$ satisfying $\pi_{A} x=0$. Note that if $x=\sum \alpha_{i} b_{i}$ relative to a basis $\left\{b_{1}, b_{2}, \cdots, b_{n}\right\}$, then $O(x)=\left(\alpha_{1}, \alpha_{2}, \cdots, \alpha_{n}\right)$. If $\sigma$ is in $\operatorname{Sp}(V)$, then the order $O(\sigma)$ of $\sigma$ is the smallest ideal $A$ satisfying $\pi_{A} \sigma$ is in Center $(\operatorname{Sp}(V / A V))$. That is, $O(\sigma)$ is the smallest ideal $A$ with $\sigma$ in $\operatorname{GSp}(V, A)^{1}$. If $G$ is a subgroup of $\operatorname{Sp}(V)$, then the order $O(G)$ of $G$ is the smallest ideal $A$ with $G \leqq \operatorname{GSp}(V, A)$.

Lemma 3.1. For the isometries in (2),

(1) $O\left(\sigma_{u, x}\right)=O(x), O\left(\sigma_{v, x}\right)=O(x)$.

(2) $O\left(\tau_{a, \lambda}\right)=O(\lambda)$.

(3) $O(\Delta)=R$.

(4) $O\left(\Phi_{\varepsilon}\right)=(\varepsilon-1)$.

THEOREM 3.2. Let $R$ be a commutative ring and $A$ be an ideal of $R$. Let $V=H \perp W$ be a symplectic space where $H=R u \oplus R v$ is a hyperbolic plane. Suppose

$$
\begin{aligned}
& x=\delta u+\eta v+\bar{x} \\
& y=\bar{\delta} u+\bar{\eta} v+\bar{y}
\end{aligned}
$$

$(\bar{x}, \bar{y}$ in $W$ ) where $x \equiv y$ modulo $A V$. If $\delta$ and $\bar{\delta}$ are units, then there is an isometry $\sigma$ in $\operatorname{SSp}(V, A)$ with $\sigma(x)=y$.

Proof. Let $z=\bar{\delta}^{-1} \bar{y}$. Then

$$
\begin{aligned}
\sigma_{v, z}(z)= & \delta u+\eta_{1} v+\left(\bar{x}-\delta \bar{\delta}^{-1} \bar{y}\right) \\
& \sigma_{v, z}(y)=\bar{\delta}+\bar{\eta} v
\end{aligned}
$$

where $\eta_{1}=\eta-\beta\left(\bar{\delta}^{-1} \bar{y}, \bar{x}\right)$. Set $q=\bar{x}-\delta \bar{\delta}^{-1} \bar{y}$. Since $x \equiv y$ modulo

1 This ideal exists due to 2.10 . 
$A V$, we have $\delta \equiv \bar{\delta}$ modulo $A$ and $\bar{x} \equiv \bar{y}$ modulo $A V$, and consequently $O(q) \subset A$. Let $\sigma_{1}=\sigma_{v, \delta^{-1} q}$. Then $O\left(\sigma_{1}\right) \subset A$, i.e., $\sigma_{1}$ is in $\operatorname{SSp}(V, A)$, and

$$
\begin{aligned}
\sigma_{1} \sigma_{v, z}(x) & =\delta u+\eta_{1} v \\
\sigma_{v, z}(y) & =\bar{\delta} u+\bar{\eta} v
\end{aligned}
$$

where $\delta$ and $\bar{\delta}$ are units. Since $\delta \equiv \bar{\delta}$ modulo $A$, we have $\delta \bar{\delta}^{-1} \equiv 1$ modulo $A$. Then

$$
\begin{aligned}
& \Phi_{\delta}^{-1} \sigma_{1} \sigma_{v, z}(x)=u+\lambda v \\
& \Phi_{\delta}^{-1} \sigma_{v, z}(y)=(1+a) u+\bar{\lambda} v
\end{aligned}
$$

where $1+a$ is a unit and $a$ is in $A$. Then, $\Phi_{1+a}^{-1}$ is in $\operatorname{SSp}(V, A)$ and

$$
\Phi_{1+a}^{-1} \Phi_{\delta}^{-1} \sigma_{v, z}(y)=u+\mu v
$$

where $\lambda \equiv \mu$ modulo $A$. Let $\lambda=\mu-\alpha$ where $\alpha$ is in $A$. Then

$$
\begin{aligned}
\sigma_{v, \alpha v}(u+\mu v) & =u+[\mu-\alpha] v \\
& =u+\lambda v
\end{aligned}
$$

where $\sigma_{v, \alpha v}$ has order $\subset A$. That is, if $\sigma=\sigma_{v, z}^{-1} \Phi_{\delta} \Phi_{1+a} \sigma_{v \alpha v}^{-1} \Phi_{\delta}^{-1} \sigma_{1} \sigma_{v, z}$ then $\sigma(x)=y$. Further, $\sigma \equiv I$ modulo $A$ and thus $\sigma$ is in $\operatorname{SSp}(V, A)$. This completes the proof.

We note that, using the calculations in 2.2, the above expression for $\sigma$ may be rewritten as a product of ESD-transvections of orders $\subset A$.

Suppose $R$ is a commutative ring with 2 a unit and 2-fold stable range one (see discussion before 2.12): Let $V=H_{1} \perp \cdots \perp H_{m}$ be a decomposition into hyperbolic planes where $H_{i}=R u_{i} \oplus R v_{i}$ for $1 \leqq i \leqq m$. Let

$$
\begin{aligned}
& u=u_{1}, \quad v=v_{1}, \\
& x=\sum_{i} \delta_{i}^{(1)} u_{i}+\sum_{i} \eta_{i}^{(1)} v_{i}, \quad \text { and } \\
& y=\sum_{i} \delta_{i}^{(2)} u_{i}+\sum_{i} \eta_{i}^{(2)} v_{i}
\end{aligned}
$$

be unimodular vectors in $V$ with $x \equiv y$ modulo $A V$. Since 2 is a unit and $x$ and $y$ are unimodular,

$$
\left(\delta_{1}^{(i)}, 2 \eta_{1}^{(i)},-\delta_{2}^{(i)}, \eta_{2}^{(i)}, \cdots,-\delta_{m}^{(i)}, \eta_{m}^{(i)}\right)=R
$$

for $i=1,2$. Since $R$ has 2-fold stable range one, it is straightforward to produce $\alpha_{1}, \alpha_{2}, \cdots, \alpha_{m}, \gamma_{2}, \cdots, \gamma_{m}$ with 


$$
\delta_{1}^{(i)}+2 \alpha_{1} \eta_{1}^{(i)}+\sum_{j}\left[\alpha_{j} \eta_{j}^{(i)}-\gamma_{j} \delta_{j}^{(i)}\right]=\mu_{i}
$$

where $\mu_{i}$ is a unit for $i=1,2$. Thus, if $w=\alpha_{1} u_{1}+\cdots+\alpha_{m} u_{m}+$ $\gamma_{2} v_{2}+\cdots+\gamma_{m} v_{m}$ then (in the notation of 3.2).

$$
\begin{aligned}
& \sigma_{u, w}(x)=\delta u+\eta v+\bar{x} \\
& \sigma_{u, w}(y)=\bar{\delta} u+\bar{\eta} v+\bar{y}
\end{aligned}
$$

where $\sigma_{u, w}(x) \equiv \sigma_{u, w}(y)$ modulo $A$ and $\delta$ and $\bar{\delta}$ are units. Combining this discussion with Theorem 3.2, we have the next result.

THEOREM 3.3. Let $R$ be commutative ring which has 2-fold stable range one and 2 anit. Let $A \neq R$ be an ideal in $R$. Let $V$ be a symplectic space over $R$ and $E=\{x$ in $V \mid x$ is unimodular $\}$. Then $\operatorname{SSp}(V, A)$ acts as a transformation group on $E$ and the $\operatorname{SSp}(V, A)$-orbits of $E$ are precisely the congruence classes of $E$ modulo $A V$.

Thus, under the hypothesis of 3.3, then for $x$ and $y$ in $E$ we have $x \equiv y$ modulo $A V$ if and only if there is a $\sigma$ in $\operatorname{SSp}(V, A)$ with $\sigma x=y$.

COROLlARY 3.4 (under the hypothesis of 3.3). The group $\operatorname{SSp}(V, A)$ acts as a transformation group on the family $\mathscr{H}$ of hyperbolic planes of $V$. Two hyperbolic planes $H$ and $\bar{H}$ are in the same $\operatorname{SSp}(V, A)$-orbit if and only if $\pi_{A} H=\pi_{A} \bar{H}$.

Proof. The proof is similar to the proof of $2.8(\mathrm{~b})$ or the proof in the local ring case given in ([13], Theorem 3.24).

CoROLlaRY 3.5 (under the hypothesis of 3.3). The group $\operatorname{SSp}(V, A)$ is generated by ESD-transvections of order contained in $A$.

Proof. The proof is analogous to the proof where $R$ is a local ring given in ([13], Theorem 4.9). The key idea is that if $x \equiv y$ modulo $A V$ then the $\sigma$ in $\operatorname{SSp}(V, A)$ with $\sigma(x)=y$ may be written as a product of ESD-transvections as was noted after Theorem 3.2

COROLLARY 3.6 (under the hypothesis of 3.3).

$$
\operatorname{SSp}(V, A)=[\operatorname{Sp}(V), \operatorname{GSp}(V, A)]=[\operatorname{Sp}(V), \operatorname{SSp}(V, A)]
$$

where $A$ is an ideal of $R$.

Proof. The case $A=R$ is given in the previous section. We 
may assume $A \neq R$. By reducing modulo $A$, it is easy to see that

$$
[\operatorname{Sp}(V), \operatorname{SSp}(V, A)] \subset[\operatorname{Sp}(V), \operatorname{GSp}(V, A)] \subset \operatorname{SSp}(V, A) .
$$

So it suffices to show that each generator of $\operatorname{SSp}(V, A)$ is in $[\operatorname{Sp}(V), \operatorname{SSp}(V, A)]$. Consider $\sigma_{u, x}$ where the $O(x) \subset A$. Select units $\varepsilon$ and $\alpha$ as in the proof of 2.12. Then $O\left(\sigma_{u, \alpha x}\right)=O\left(\sigma_{u, x}\right) \subset A$ and, as in 2.12 ,

$$
\sigma_{u, x}=\left[\Phi_{\varepsilon}, \sigma_{u, \alpha x}\right] ;
$$

thus, $\sigma_{u, x}$ is in $[\operatorname{Sp}(V), \operatorname{SSp}(V, A)]$.

\section{REFERENCES}

1. H. Bass, Algebraic K-theory, Benjamin New York, 1968.

2. — K-theory and stable algebra, Publ. I.H.E.S., 22 (1964), 5-60.

3. I. G. Connell, Some ring theoretic Schröder-Bernstein Theorem, Trans. Amer. Math. Soc., 132 (1968), 335-351.

4. D. Estes and J. Ohm, Stable range in commutative rings, J. Algebra, 7 (1967), 343-362.

5. K. R. Goodearl, Von Neumann Regular Rings, Pitman London, 1979

6. W. Jehne, Die Struktur der symplektischen Gruppen über lokalen und dedekindschen Ringen, Sitzungber, Heidelberg Akad. Wiss. Math. Naturwiss, 3 (1962/64), 189-235.

7. W. van der Kallen, The $K_{2}$ of rings with many units, Ann. Scient. Ec. Norm. Sup., 10 (1977), 473-515.

8. F. Kirchheimer, Die Normalteiler der symplektischen Gruppen über beliebigen lokalen Ringen, J. Algebra, 50 (1978), 228-241.

9. B. Kirkwood and B. McDonald, The Witt ring of a full ring, J. Algebra, 64 (1980), 148-166.

10. - The orthogonal group over a full ring, to appear J. Algebra.

11. W. Klingenberg, Symplectic groups over local rings, Amer. J. Math., 85 (1963), 232-240.

12. V.I. Kopeyko, The stablization of symplectic groups over a polynomial ring, Math. USSR Sbornik, 34 (1978), 655-669.

13. B. R. McDonald, Geometric Algebra Over Local Rings, Marcel Dekker, Inc., New York, 1976.

14. B. McDonald and B. Hershberger, The orthogonal group over a full ring, J. Algebra, 51 (1978), 536-549.

15. B. McDonald, $G L_{2}$ of rings with many units, Comm. in Algebra, 8(9) (1980), 869-888.

16. - The special orthogonal group over a full ring, J. Algebra.

17. L. McQueen and B. R. McDonald, Automorphisms of the symplectic group over a local ring, J. Algebra, 30 (1974), 485-495.

18. E. F. Robertson, Some properties of a subgroup of $\operatorname{Sp}_{\Omega}(R)$, J. London Math. Soc., (2) 4 (1971), 65-78.

19. Yu. V. Sosnovskii, Commutator structure of symplectic group, translated from Matematicheskie Zametki, 24 (1978), 641-648.

20. J. T. Stafford, Stable structure of noncommutative Noetherian rings, J. Algebra, 47 (1977), 244-267.

21. R. Warfield, Notes on cancellation, stable range and related topics, 1975.

$22 .-$ Stable equivalence of matrices and resolutions, Comm. in Algebra, 6 (17), (1978), 1811-1828.

23. - Stable generation of modules, Module Theory, Springer-Verlag Lecture 
Notes \#700 (Edited: C. Faith, S. Wiegand) (1979), 16-33.

24. R. B. Warfield, Cancellation of modules and groups and stable range of endomorphism rings, to appear.

Received December 6, 1979 and in revised form May 14, 1980. The second author was partially supported by N.S.F. Grant No. MCS-7902573.

SWeet Briar College

SWEET BRIAR, VA 24595

AND

The University of OKLAHOMA

NORMAN, OK 73019 



\section{PACIFIC JOURNAL OF MATHEMATICS}

\section{EDITORS}

DONALD BABBITT (Managing Editor)

University of Galifornia

Los Angeles, California 90024

Hugo RossI

University of Utah

Salt Lake City, UT 84112

C. C. MOORE AND ANDREW OGG

University of California

Berkeley, CA 94720
J. DUGUNDJI

Department of Mathematics University of Southern California Los Angeles, California 90007

R. Finn AND J. Milgram Stanford University Stanford, California 94305

\section{ASSOCIATE EDITORS}

R. ARENS

E. F. BECKENBACH

B. H. NEUManN

F. WOLF

K. YOSHIDA

\section{SUPPORTING INSTITUTIONS}

UNIVERSITY OF ARIZONA

UNIVERSITY OF BRITISH COLUMBIA CALIFORNIA INSTITUTE OF TECHNOLOGY

UNIVERSITY OF CALIFORNIA

MONTANA STATE UNIVERSITY

UNIVERSITY OF NEVADA, RENO

NEW MEXICO STATE UNIVERSITY

OREGON STATE UNIVERSITY
UNIVERSITY OF OREGON

UNIVERSITY OF SOUTHERN CALIFONIA

STANFORD UNIVERSITY

UNIVERSITY OF HAWAII

UNIVERSITY OF TOKYO

UNIVERSITY OF UTAH

WASHINGTON STATE UNIVERSITY

UNIVERSITY OF WASHINGTON 


\section{Pacific Journal of Mathematics}

\section{Vol. 92, No. $1 \quad$ January, 1981}

Michael E. Adams and J. Sichler, Lattices with unique complementation . ....1

Walter Allegretto, Positive solutions and spectral properties of second order

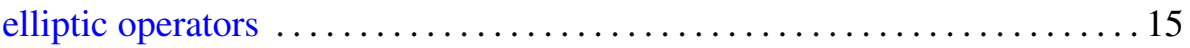

Philip J. Boland and Sean Dineen, Holomorphy on spaces of distribution . . 27

Duncan Alan Buell, Philip A. Leonard and Kenneth S. Williams, Note on

the quadratic character of a quadratic unit $\ldots \ldots \ldots \ldots \ldots \ldots \ldots \ldots \ldots$

Herbert Busemann and Bhalchandra B. Phadke, Two theorems on

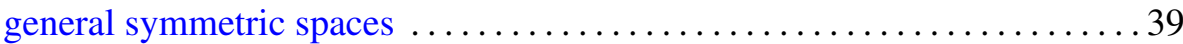

Emeric Deutsch, Bounds for the Perron root of a nonnegative irreducible

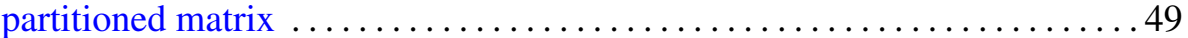

Charles F. Dunkl, A difference equation and Hahn polynomials in two

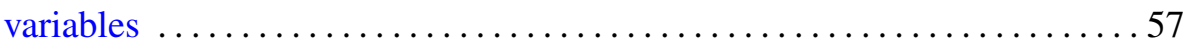

Gustave Adam Efroymson, The Riemann mapping theorem for planar Nash

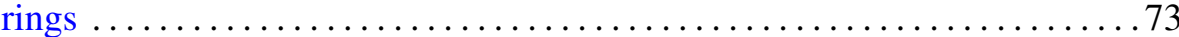

John Albert Fridy and Robert Ellis Powell, Tauberian theorems for matrices generated by analytic functions

Denton Elwood Hewgill, John Hamilton Reeder and Marvin Shinbrot,

Some exact solutions of the nonlinear problem of water waves .......887

Bessie Hershberger Kirkwood and Bernard Robert McDonald, The

symplectic group over a ring with one in its stable range

Esther Portnoy, Transitive groups of isometries on $H^{n}$

Jerry Ridenhour, On the sign of Green's functions for multipoint boundary

value problems

Nina M. Roy, An $M$-ideal characterization of $G$-spaces

Edward Barry Saff and Richard Steven Varga, On incomplete

polynomials. II

Takeyoshi Satō, The equations $\Delta u=P u(P \geqq 0)$ on Riemann surfaces and

isomorphisms between relative Hardy spaces

James Henry Schmerl, Correction to: "Peano models with many generic classes"

Charles Madison Stanton, On the closed ideals in $A(W)$

Viakalathur Shankar Sunder, Unitary equivalence to integral operators

Pavel G. Todorov, New explicit formulas for the $n$th derivative of composite functions

James Li-Ming Wang, Approximation by rational modules on boundary sets

Kenneth S. Williams, The class number of $Q(\sqrt{p})$ modulo 4 , for $p \equiv 5$

$(\bmod 8)$ a prime 